\title{
Imaging beyond the coverslip
}

\author{
Technical advances provide practical options for imaging deep within living tissues and \\ organisms.
}

Imaging biological structures and processes as they occur in vivo is a long-standing goal of optical microscopy, with enormous implications for understanding fundamental aspects of life-from the structure and function of the nervous system to how cancers form, grow and metastasize. Our desire to peer into the inner workings of the body to better understand it is an old one. Centuries ago, van Leeuwenhoek carried out intravital microscopy to examine how blood flows within tissues (described in The Select Works of Antony van Leeuwenhoek, Containing His Microscopical Discoveries in Many of the Works of Nature by Samuel Hoole; G. Sidney, London, 1800). Given the hundreds of intervening years of optimization, one could imagine that in vivo imaging is a solved problem, and indeed methods for imaging very small, transparent organisms, like zebrafish embryos, work quite well. However, acquiring images in most living tissues still presents myriad challenges for optical microscopy.

These have mainly to do with the nature of the samples themselves and their interaction with light. Tissues and most organisms, even relatively small ones like Drosophila embryos, are turbid and interact with both the light used to excite fluorophores within the sample and the emitted light leaving the sample. These interactions mainly take the form of light scattering and absorption, and function to weaken and blur signals used to generate images. Additional challenges can be caused by refractive index heterogeneity in tissues, which can distort wavefronts and compromise image quality. As a consequence imaging of nontransparent tissues with visible light has largely been limited to regions near the surface.

In this Focus issue, we bring together papers describing innovations in optical microscopy that help overcome the barriers for imaging deeply within living organisms and tissue. These advances have enabled a wide range of experimental observations, from imaging of fly, fish and mouse development to volumetric functional imaging in the mouse cortex.

A Review by Weijian Yang and Rafael Yuste (p349) highlights advances in microscopy that have enabled neuronal function to be imaged in vivo; a Perspective by Na Ji (p374) introduces adaptive optics to correct aberrations in biological imaging; and a Review by Rory Powers and Jan Huisken (p360) describes considerations for building and using an instrument for light-sheet microscopy, a technique that has provided unparalleled imaging of developmental biology. Although many of

the methods require specialized equipment, especially those at the very cutting edge, each piece offers practical guidance for experimentalists who do not necessarily specialize in optics. In addition, two research papers in this issue report approaches for improved neuronal imaging. Ouzonov and colleagues (p388) describe improvements to three-photon microscopy that enable calcium imaging in the hippocampus of living mice, and Tank and colleagues (p420) report a method for faster volumetric functional brain imaging.

Although these pieces cover a huge breadth of methodological research, it would be impossible to include the entire range of in vivo approaches. For example, wavefront shaping methods developed specifically for dealing with light scattering are not discussed here in detail. Nor are intravital imaging approaches that use surgical windows to image specific tissues in animals or endoscopic methods that bring lenses closer to the region to be imaged. We also omit the field of tissue clearing in this issue; clearing methods are powerful and are seeing wide adoption among biologists, but are incompatible with living tissue.

Other crucial players described only tangentially in this issue are the probes necessary for high-quality fluorescence microscopy. Although GFP and GFPbased probes still largely dominate many aspects of in vivo imaging, interest has shifted toward probes that excite and emit in the red to near-infrared region of the spectrum. This is because such light is able to penetrate more deeply into tissues, as it is not strongly absorbed by endogenous pigments, biomolecules or water. Brighter and more photostable forms of these probes are poised to advance deep tissue imaging.

Finally, while the papers in this issue focus on optical microscopy, methods such as photoacoustic and ultrasound imaging are better suited than fluorescence microscopy for in vivo imaging at centimeter depths and beyond, and especially for imaging deep within intact mammals. These methods are still rapidly advancing, are largely complementary to optical microscopy, and should see broader adoption in years to come.

To round out this Focus, we have curated a collection of manuscripts published in Nature Research journals that highlight recent technical advances in deep imaging of live tissues (www.nature.com/livedeepimaging). We hope that these resources provide a starting point for discovering deep tissue imaging methods and encourage users to try these techniques themselves. 